\title{
FXIIIA and TGF- $\beta$ over-expression produces normal musculo-skeletal phenotype in TG2-/- mice
}

\author{
U. Tarantino $\cdot$ F. Oliva $\cdot$ G. Taurisano $\cdot$ A. Orlandi $\cdot$ \\ V. Pietroni $\cdot$ E. Candi $\cdot$ G. Melino $\cdot$ N. Maffulli
}

Received: 20 February 2008/Accepted: 15 April 2008/Published online: 2 July 2008

(C) Springer-Verlag 2008

\begin{abstract}
Transglutaminase (TGs) enzymes and proteins crosslinking have for long time been implicated in the formation of hard tissue development, matrix maturation and mineralization. Among the TGs family members, in the context of connective tissue formation, TG2 and Factor XIII are expressed in cartilage by hypertrophic chondrocytes. Here, we analyse the morphological consequences of TG2 deficiency, during the development of skeletal elements. When TG2 is absent, there are not gross abnormalities in the development of the skeletal system, probably from compensatory mechanisms resulting in increased expression of FXIIIA and TGF- $\beta$ 1. In vivo other TGs may be involved in promoting chondrocytes and osteoblast differentiation and matrix mineralisation
\end{abstract}

Keywords Endochondral ossification - Transglutaminase

U. Tarantino $\cdot$ F. Oliva $(\varangle) \cdot$ G. Taurisano

Department of Orthopaedics and Traumatology,

University of Rome "Tor Vergata", Viale Oxford 81, 00133 Rome, Italy

e-mail: olivafrancesco@hotmail.com

\section{A. Orlandi}

Department of Anatomic Pathology,

University of Rome "Tor Vergata", Viale Oxford 81,

00133 Rome, Italy

\section{Pietroni - E. Candi - G. Melino}

Department of Experimental Medicine and Biochemical

Sciences, University of Rome "Tor Vergata", via Montpellier 1, 00133 Rome, Italy

\section{N. Maffulli}

Department of Trauma and Orthopaedic Surgery,

Keele University School of Medicine,

Hartshill, Thornburrow Drive, Stoke-on-Trent,

Staffordshire ST4 7QB, UK

\section{Introduction}

The process of endochondral bone development in vertebrates is characterised by replacement of a cartilage anlagen by bone: chondrogenesis and osteogenesis are tightly coordinated in time and space (Erlebacher et al. 1995; Marks and Hermey 1996). Three tissues appear to be the main players in the initiation of endochondral ossification: the cartilage, the adjacent perichondrium, and the invading vasculature. Interactions among these tissues are synchronised, and a large number of secreted and intracellular factors acting in this process have been recently identified (Colnot 2005). Hypertrophic growth plate chondrocytes provide a scaffold for subsequent formation of trabecular bone by mineralising their surrounding matrix and inducing a bone collar, the precursor of cortical bone, in the adjacent perichondrium (Chung and Lanske 1998). In mice, endochondral ossification starts at 14.5-day embryo. By 19-day embryo, ossification is complete (Wirtschafter 1966).

Transglutaminase (TGs) enzymes and proteins crosslinking have been implicated in the formation of hard tissue, matrix maturation and mineralisation (Lorand and Graham 2003; Aeschlimann et al. 1993, 1996; Aeschlimann and Thomazy 2000; Candi et al. 2005; Melino et al. 2000). TGs are a nine member family of calcium-dependent enzymes which catalyse the formation of covalent $\gamma$-(glutamyl)-e-lysyl bonds (isopeptide bonds or crosslinks) among protein-bound lysines and glutamines, thus forming large polymers or proteins. TGs can also use as substrate primary amines (putrescine, spermine and spermidine) to specific glutamine residues (Lorand and Graham 2003). TGs crosslinking activity can take place in the cytosol of the cells, at the cells surface, and/or the bone extracellular matrix compartments (Lorand and Graham 2003). 
Many TG substrates directly or indirectly related to matrix maturation and mineralisation have been identified in vitro so far, including collagen I-V-X-XI, fibronectin, osteopontin, osteonectin and bone sialoprotein (Mosher and Schad 1979; Jeong et al. 1995; Kaartinen et al. 1999; Lorand et al. 1998; Turner and Lorand 1989; Prince et al. 1991; Sorensen et al. 1994; Kaartinen et al. 2002; Esposito and Caputo 2005; Johnson 2007). However, the function of the crosslinking is not yet understood. Among the TGs family members, in the context of connective tissue formation, TG2 and Factor XIII are expressed in cartilage by hypertrophic chondrocytes (Aeschlimann et al. 1996; Aeschlimann and Thomazy 2000; Nurminskaya and Linsenmayer 2002; De Laurenzi and Melino 2001; Nurminskaya et al. 1998). In addition, several reports correlate in vitro TG2 expression, chondrocyte differentiation and matrix mineralization (Aeschlimann et al. 1996; Aeschlimann and Thomazy 2000; Nurminskaya et al. 1998; Nurminskaya and Linsenmayer 2002; Johnson and Terkeltaub 2005). Also, TGF- $\beta$ family is involved during endochondral ossification and bone remodelling (Janssens et al. 2005). However, the mechanisms behind these phenomena mediated by TGs, and in particular those mediated by TG2, are not known.

We characterised the morphological consequences of TG2 deficiency produced by a gene-targeting approach in mice (De Laurenzi and Melino 2001) during the development of skeletal elements.

\section{Materials and methods}

The local Animal Experiment Committee approved all procedures performed in this study.

Tissue preparation

Five newborns mice of TG2 knock-out and wild type were stained with $0.5 \%$ alizarin red and $0.015 \%$ alcian blue were performed as described previously (Hogan et al. 1994).

Five skeletons of 13.5- to 18.5-day embryo of TG2 knock-out and wild type mice, plus five newborn mice of TG2 knock-out and wild type mice were euthanised with an overdose of chloroform (total numbers of animals 70). After some skin incisions, embryos were processed. All samples were fixed in $10 \%$ buffered formalin for 12 hours, and decalcified for 48 hours in Decalcifier II (Surgipath Medical Industries, Richmond, IL, USA). After usual processing and paraffin embedding, $5 \mu \mathrm{m}$ section were placed on SuperFrost Plus glass slides (Menzel-Gläser, Braunschweig, Germany) and used for haematoxylin-eosin or immunohistochemical stainings. Femurs were selected for this study.
Immunohistochemical study

Slides were heated in a dry oven overnight at $60^{\circ} \mathrm{C}$, de-paraffinised in xylene, and rehydrated in graded concentrations of ethanol. Endogenous peroxidase activity was blocked by incubation in $0.3 \%$ hydrogen peroxide and methanol. Slides were re-hydrated in phosphate-buffered saline. Non-specific antibody binding was blocked by incubation with normal goat serum (Dako Cytomation, Glostrup, Denmark). Optimal anti-TG2 antibody (Covalab, Vinci-Biochem, Firenze, Italy) dilution was found to be 1:75 for $30 \mathrm{~min}$. Slides were then incubated with a biotinlabeled goat anti-rabbit secondary antibody (Dako Cytomation, Glostrup, Denmark), followed by a streptavidinhorseradish peroxidase conjugate. Bound antibody was revealed with the use of the substrate $3,3^{\prime}$-diaminobenzidine. Sections were counterstained with haematoxylin, washed, dehydrated with graded concentrations of ethanol, cleared in xylene, mounted, and examined at light microscopy. Human breast carcinoma was included with each batch of sections as a positive control. The location of the TG2 was determined by comparing each immunohistochemical section with the adjacent slice stained with haematoxylin-eosin.

\section{Western blotting}

Western Blots were performed using the whole sample derived from a mouse femur, lysed in Laemli Buffer. The blots (polyvinylidene difluoride membrane) were kept in blocking solution (10\% dried milk) for $2 \mathrm{~h}$ at room temperature. Blots were incubated shaking for $2 \mathrm{~h}$ at room temperature with the antibodies to TG2 (dilution 1:500, Covalab); anti-FXIII (dilution 1:1,000, Santa Cruz); anti-TGF $\beta 1$ (dilution 1:500, Santa Cruz); anti- $\beta$ tubulin (dilution 1: 1,000, Santa Cruz). After three washes in PBS-tween $20(0.05 \%)$, secondary antibody peroxidase conjugated (dilution 1:10,000) was added in blocking solution, shaking for $1 \mathrm{~h}$ at room temperature. Proteins were detected using the enhanced chemiluminescence method (ECL, Amersham).

\section{Results}

The size of the skeletal elements and the relative ratio of cartilage to bone in TG2-deficient mice were indistinguishable from those of new born wild-type animals (Fig. 1a-c). Haematoxylin-eosin stained sections showed that the wild-type fetal growth plate consists of three major layers of chondrocytes: proliferating, prehypertrophic, and hypertrophic layers. The proliferating layer contained two distinct types of chondrocytes. Proliferating chondrocytes 

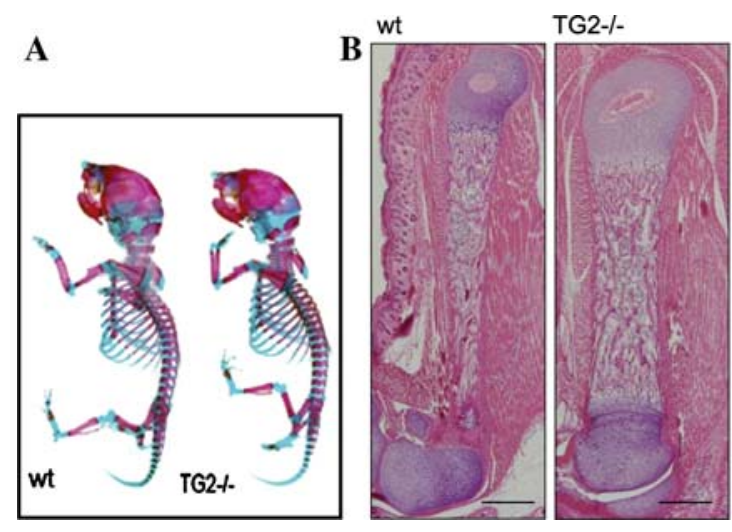

Fig. 1 Cartilage and bone develop normally in newborns lacking TG2. The skeletons from newborn littermates of wild-type and TG2-/were stained with alcian blue and alizarin red, detecting cartilage and bony structures, respectively (a). Haemotoxylin-eosin stained sections of femur of new born wild type and TG2-/- mice (b). Analysis of histological embryos femurs development from wild type and TG2-/mice from 13.5 to 18.5 days stained with haematoxylin-eosin (c). The

close to the articular surface were round and randomly scattered, with the typical features of peri-articular chondrocytes. Proliferating chondrocytes immediately below the peri-articular proliferating chondrocytes were flat, and formed longitudinal columns (Fig. 2a, b): they were columnar chondrocytes.

TG2-/- animals were indistinguishable in size and behavior from wild-type mice in all the embryonic stages analysed, and up to an age of 1 year (data not shown). Adult mice lacking TG2 displayed no obvious skeletal abnormalities, and no skeletal elements were missing or deformed. Immunohistochemistry confirmed the expression of the TG2 in the hypertrophic chondrocytes of wild type mice, and its absence in knock-out mice (Fig. 2c, d). In knock-out mice, deficiency of TG2 causes no obvious consequences during embryonic skeletal development and in adulthood. The observation that TG2-/- mice have no overt skeletal phenotype, suggest that at least a second TG enzyme must influence matrix assembly and mineralisation. Previously, it has been described that, in addition to TG2, cultured osteoblasts also express the plasma transglutaminase Factor XIIIA (FXIIIA; Nurminskaya and Linsenmayer 2002; De Laurenzi and Melino 2001; Nurminskaya and Kaartinen 2006). To verify whether Factor XIIIA is involved, we analysed by western-blot its expression on protein extracts obtained from wild type and TG2-/- femurs (Fig. 3). The distal femoral epiphysis at the 18.5 day post conception of wild type and TG2-/- mice were isolated, and proteins were extracted as indicated in "Materials and methods". Western blot analysis indicate that FXIIIA protein is increased of about fivefold (Fig. 3), as evaluated by densitometry analysis normalised over the

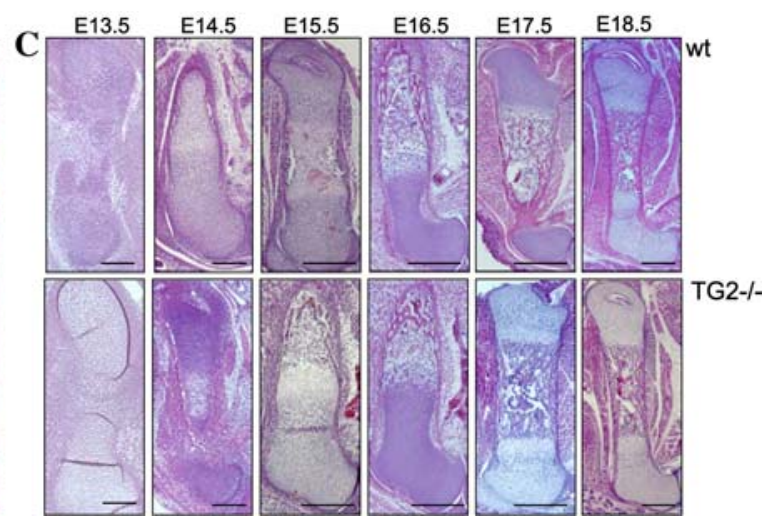

primary ossification centers and periosteal cuffs are fully formed. The secondary ossification center of the proximal epiphysis is already visible at the 14.5 days in the knock-out embryo, and fully formed in both the panels at day 18.5. Bars $200 \mu \mathrm{m}$ for wild type and TG2-/- at E13.5 and E14.5. Bars $250 \mu \mathrm{m}$ for wild type and TG2-/- at E15.5, E16.5 and E17.5, bars $500 \mu \mathrm{m}$ for wild type and TG2-/- at E18.5

loading control ( $\beta$-tubulin). We also determined the levels of transforming growth factor beta-1 (TGF- $\beta 1$ ). Western blot analysis showed a marked increase of the TGF- $\beta 1$ protein level in TG2-/- bones as compared with wild type (Fig. 3).

\section{Discussion}

TG2 and Factor XIIIA are the only identified transglutaminases in human, mouse, and chick growth plates. These locations led to speculation that transglutaminases regulate endochondral ossification in particular both terminal cell differentiation and matrix calcification (Johnson 2007; Nurminskaya and Linsenmayer 2002; Nurminskaya et al. 2002; Johnson et al. 2001) also FXIIIA-null mice show a dramatic decrease in COL I deposition in osteoblast cultures (Al-Jallad et al. 2006). Indeed, many investigators confirmed this hypothesis in vitro, but to our knowledge no studies on the endochondral ossification of TG2-/- mice during are available.

Recently, a $37 \mathrm{kDa}$ form of Factor XIIIA was discovered. It is located exclusively in cartilage and bone, and not in plasma. This shortened form of Factor XIIIA may be controlling mineralisation or have unique functions (Nakano et al. 2007), similar to those of the shortened isoform of TG2 in brain (Antonyak et al. 2006).

Latent TGF binding protein-1 is particularly interesting because it release the active TGF- $\beta 1$ only after its TG2catalysed linkage to the matrix (Nunes et al. 1997). This cytokine affects many aspects of bone formation (Janssens et al. 2005), and TGs may regulate the extracellular levels 
Fig. 2 Haematoxylin-eosin staining of sections of wild type (a) and TG2-/- (b) proximal femoral growth plates from the 18.5-day embryo. Brackets indicate the approximate lengths of the growth plates.

Periarticular proliferating chondrocytes (ppc), columnar proliferating chondrocytes (cpc), prehypertrophic and hypertophic chondrocytes (p\&hc) in the growth plate are regularly arranged both in wild type anf TG2-/- samples. Bars $500 \mu \mathrm{m}$. Expression of TG2 in proximal femur of wild type (c) and TG2-/- (d) mice. Immunohistochemistry of proximal femoral growth plate sections from 18.5-day embryo. Note in the wild type the increased expression of TG intracellularly and in the interterritorial matrix of columnar proliferating chondrocytes. Bars $500 \mu \mathrm{m}$

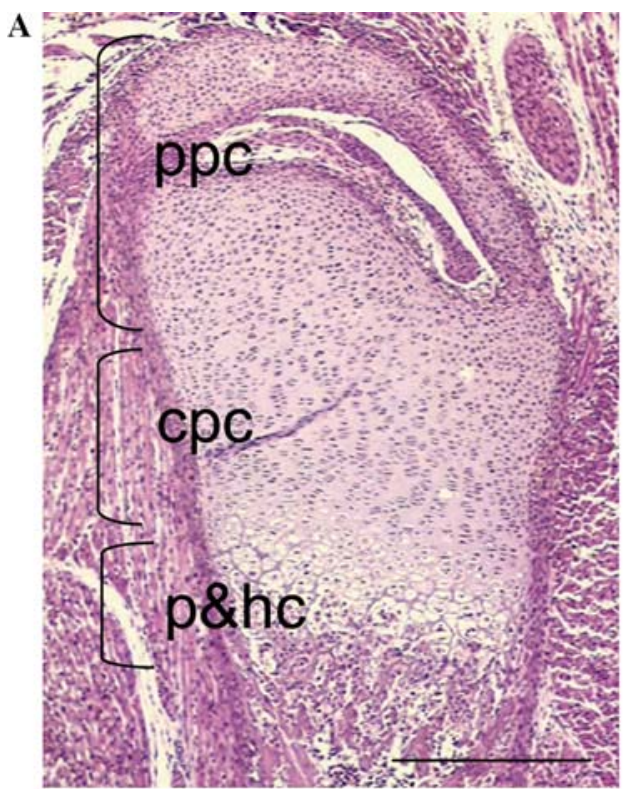

B

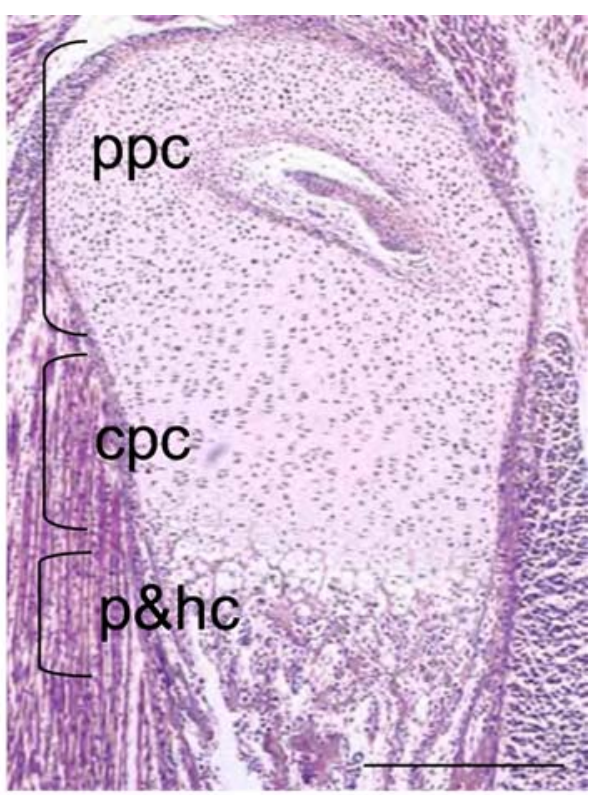

C

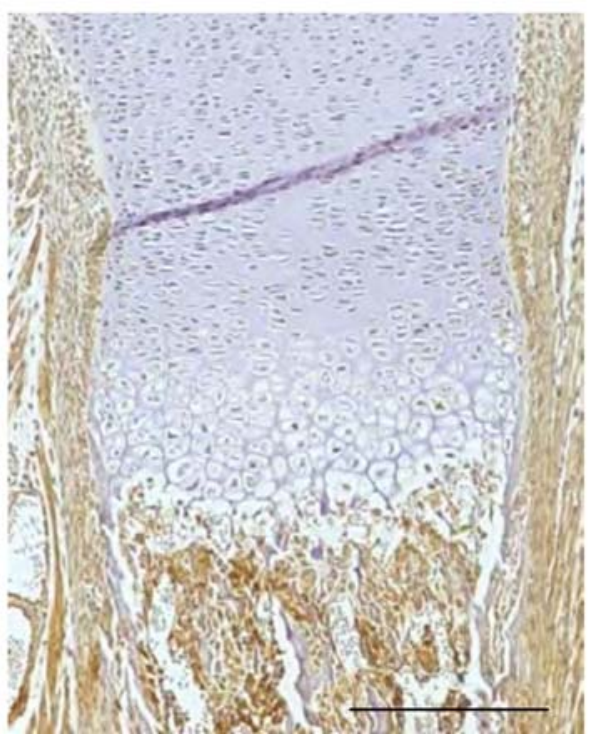

D

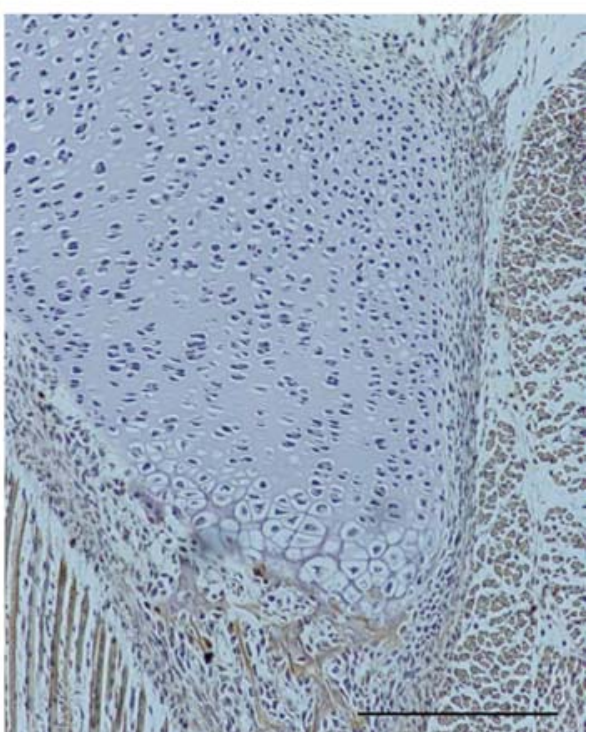

of TGF- 3 . In addition, TGF- $\beta 1$ null mice show severe skeletal defects (Geiser et al. 1998).

TGF- $\beta$ is expressed in chondrocytes, osteoblast and osteocytes (Janssens et al. 2005). Other studies have shown that low levels $(5-200 \mathrm{pg} / \mathrm{ml})$ of TGF- 32 promoted mineralisation in the co-cultures, but they demonstrated a slightly lower level of activated latent TGF- $\beta$ after treatment with recombinant TG2. This suggests that the mechanism of TG2 induced mineralisation did not involve TGF-B (Nurminskaya et al. 2003).

To study in vivo the involvement of TG2 in skeletal development, and to exclude possible developmental delay or deformities, we focused on the early development of skeletal structures in embryos from the stage embryo 13.5 to newborn.
Proliferating chondrocytes undergo a limited number of divisions and then lose the capacity by beginning to hyperthrophy. This switch is regulated by parathyroid hormone-related peptide (Chung and Lanske 1998). Prehyperthrophic and hypertophic chondrocytes increase dramatically their height, volume and matrix mass, and become more rounded until they have an egg like configuration (Fig. 2a, b).

In this study, we demonstrated no gross skeletal abnormalities or developmental delay in bone formation in the absence of TG2 in the femora of mice. Therefore, in vivo other TGs may be involved in promoting chondrocytes and osteoblast differentiation and matrix mineralisation. Among the TGs, FXIIIA has been described to contribute to TGs activity observed in both hypertrophic chondrocytes and 

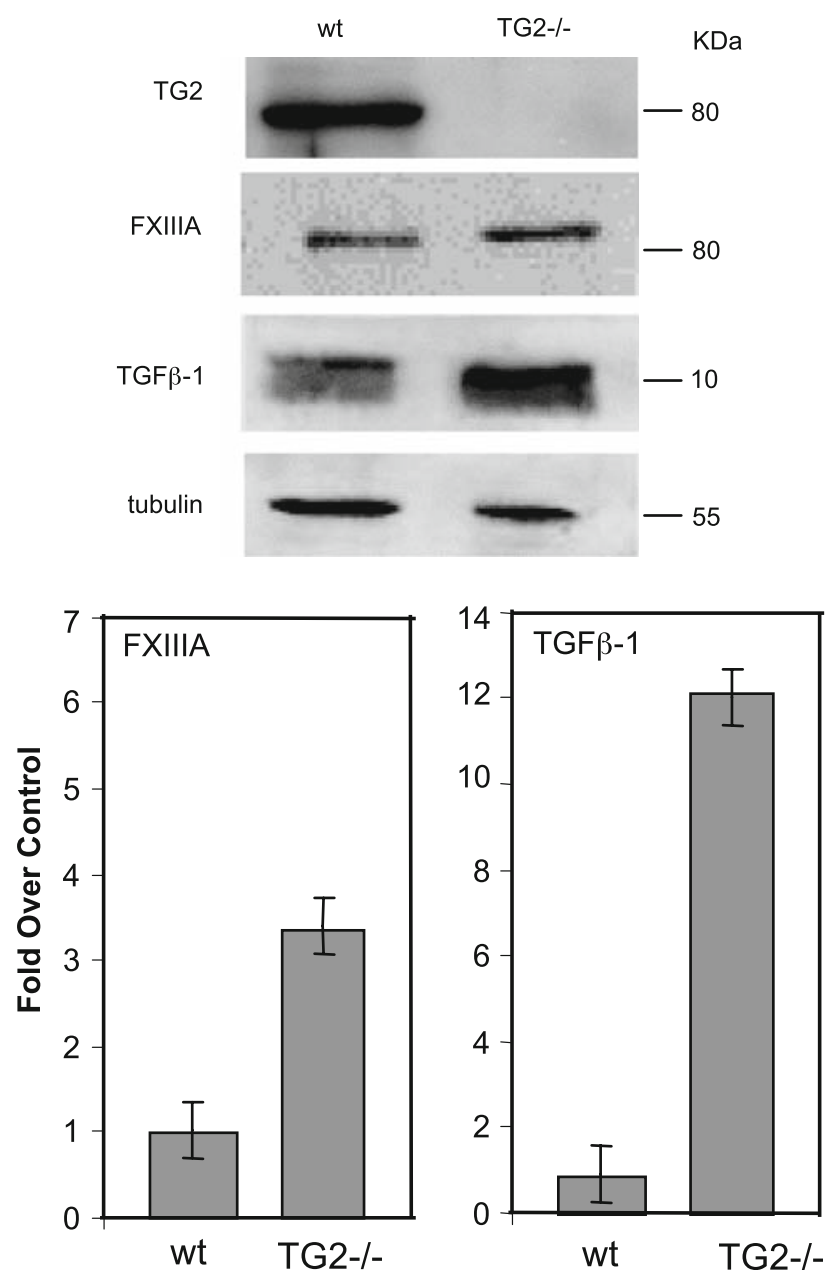

Fig. 3 Expression of FXIII and TGF- $\beta 1$ in femora of wild type and TG2-/- mice. Western blot analysis was performed on isolated distal femoral epiphysis at 18.5 days (a), with $\beta$-tubulin as loading control. The densitometric analysis (b) shows the relative expression of FXIIIA and TGF- $\beta 1$. A representative experiment of three is shown. The analysis was normalized over the loading control

osteoblasts. Interestingly, protein level of FXIIIA increases fivefold in protein extracted by TG2-/- femurs compared with wild type. Therefore, FXIIIA is expressed in vivo in bone, and we suggest that TG2 and FXIIIA work sinergically in these processes. In addition, we observed an increased level of TGF- 31 protein in TG2-/- bones in comparison with wt. TGF- $\beta 1$ is a potent multifunctional regulators of osteoblast differentiation, and TGF- $\beta 1$ activation and upregulation could be another parallel compensatory mechanism responsible for the absence of abnormal skeletal phenotype in TG2-/- mice. Therefore, results obtained in vitro can contradict in vivo observations.

In the absence of TG2, we were not able to detect phenotypical abnormalities in the femora of embryonic, young, and adult TG2-/- mice. This may result from compensatory mechanisms involving increased expression of FXIIIA and TGF- $\beta 1$.
Several questions remain open and are subject to future research. For example, are there other TG isoforms supplying the TG2 activity during endochondral ossification? Are the recent short forms of FXIIIA and TG2 in vivo the truly protagonist of endochondral ossification? Which mechanisms activate in vivo the short forms of FXIIIA and TG2? Are other parallel mechanisms involving TGs and time depending cytokines and/or growth factors important in mineralisation and stabilisation of extracellular matrix during endochondral ossification? The development of further knock-out mice for TG2, Factor XIIIA and TGF-ß 1 may help answer at least some of these questions.

Acknowledgments We are grateful to Drs Maurizio Mattei, Chiara Focaccetti, Carla Montesano for their helpful discussions and for technical assistance. This study was partially financed by Fondi di Ricerca University of Rome "Tor Vergata" to UT. The work was supported by grants from Telethon and PRIN 2006 to EC; FIRB, MIUR MinSan to GM.

\section{References}

Aeschlimann D, Mosher D et al (1996) Tissue transglutaminase and factor XIII in cartilage and bone remodeling. Semin Thromb Hemost 22:437-443

Aeschlimann D, Thomazy V (2000) Protein crosslinking in assembly and remodelling of extracellular matrices: the role of transglutaminases. Connect Tissue Res 41:1-27

Aeschlimann D, Wetterwald A et al (1993) Expression of tissue transglutaminase in skeletal tissues correlates with events of terminal differentiation of chondrocytes. J Cell Biol 120:14611470

Al-Jallad HF, Nakano Y et al (2006) Transglutaminase activity regulates osteoblast differentiation and matrix mineralization in MC3T3-E1 osteoblast cultures. Matrix Biol 25:135-148

Antonyak MA, Jansen JM et al (2006) Two isoforms of tissue transglutaminase mediate opposing cellular fates. Proc Natl Acad Sci USA 103:18609-18614

Candi E, Schmidt R et al (2005) The cornified envelope: a model of cell death in the skin. Nat Rev Mol Cell Biol 6:328-40

Chung UI, Lanske B (1998) The parathyroid hormone/parathyroid hormone-related peptide receptor coordinates endochondral bone development by directly controlling chondrocyte differentiation. Proc Natl Acad Sci USA 95:13030-13035

Colnot C (2005) Cellular and molecular interactions regulating skeletogenesis. J Cell Biochem 95:688-697

De Laurenzi V, Melino G (2001) Gene disruption of tissue transglutaminase. Mol Cell Biol 21:148-55

Erlebacher A, Filvaroff EH et al (1995) Toward a molecular understanding of skeletal development. Cell 80:371-378

Esposito C, Caputo I (2005) Mammalian transglutaminases. Identification of substrates as a key to physiological function and physiopathological relevance. FEBS J 272:615-663

Geiser AG, Zeng QQ et al (1998) Decreased bone mass and bone elasticity in mice lacking the transforming growth factor- $\beta 1$ gene. Bone 23:87-93

Hogan B, Beddington R et al (1994) Manipulating the mouse embryo: a laboratory manual. Cold Spring Harbor Laboratory, Cold Spring Harbor, New York

Janssens K, Ten Dijke P et al (2005) Transforming growth factorbeta1 to the bone. Endocr Rev 26:743-774 
Jeong JM, Murthy SN et al (1995) The fibronectin-binding domain of transglutaminase. J Biol Chem 270:5654-5658

Johnson KA (2007) Transglutaminase modification of the matrix through promotion of calcification. Curr Opin Orthop 18:467471

Johnson KA, Terkeltaub RA (2005) External GTP-bound transglutaminase 2 is a molecular switch for chondrocyte hypertrophic differentiation and calcification. J Biol Chem 280:15004-15012

Johnson KA, Hashimoto $S$ et al (2001) Interleukin-1 induces promineralizing activity of cartilage tissue transglutaminase and factor XIIIa. Am J Pathol 159:149-163

Kaartinen MT, Pirhonen A et al (1999) Cross-linking of osteopontin by tissue transglutaminase increases its collagen binding properties. J Biol Chem 274:1729-1735

Kaartinen MT, EL-Maadawy S et al (2002) Tissue transglutaminase and its substrates in bone. J Bone Miner Res 17:2161-2173

Lorand L, Graham RM (2003) Transglutaminases: crosslinking enzymes with pleiotropic functions. Nat Rev Mol Cell Biol 4:140-156

Lorand L, Dailey JE, Turner PM (1998) Fibronectin as a carrier for the transglutaminase from human erythrocytes. Proc Natl Acad Sci USA 85:1057-1059

Marks SC, Hermey DC (1996) The structure and development of bone. In: Bilezikian JP, Raisz LG, Rodan GA (eds) Principles of bone biology. Academic Press, San Diego, pp 3-14

Melino G, Candi E et al (2000) Assays for transglutaminases in cell death. Meth Enzymol 322:433-472

Mosher DF, Schad PE (1979) Cross-linking of fibronectin to collagen by blood coagulation factor XIIIA. J Clin Invest 64:781-787

Nakano Y, Al-Jallad HF et al (2007) Expression and localization of plasma transglutaminase factor XIIIA in bone. J Histochem Cytochem 55:675-685
Nunes I, Gleizes P-E et al (1997) Latent transforming growth factor- $\beta$ binding protein domains involved in activation and transglutaminase dependent cross-linking of latent transforming growth factor-b. J Cell Biol 136:1151-1163

Nurminskaya MV, Kaartinen MT (2006) Transglutaminases in mineralized tissues. Front Biosci 11:1591-1606

Nurminskaya MV, Linsenmayer TF (2002) Immunohistological analysis of transglutaminase factor XIIIA expression in mouse embryonic growth plate. J Orthop Res 20:575-578

Nurminskaya MV, Magee C et al (1998) Plasma transglutaminase in hypertrophic chondrocytes: expression and cell-specific intracellular activation produce cell death and externalization. J Cell Biol 142:1135-1144

Nurminskaya MV, Recheis B et al (2002) Transglutaminase factor XIIIA in the cartilage of developing avian long bones. Dev Dyn 223:24-32

Nurminskaya MV, Magee C et al (2003) Chondrocyte-derived transglutaminase promotes maturation of preosteoblasts in periosteal bone. Dev Biol 263:139-152

Prince CW, Dickie D, Krumdieck CL (1991) Osteopontin, a substrate for transglutaminase and factor XIII activity. Biochem Biophys Res Commun 177:1205-1210

Sorensen ES, Rasmussen LK et al (1994) Localization of transglutaminase-reactive glutamine residues in bovine osteopontin. Biochem J 304:13-16

Turner PM, Lorand L (1989) Complexation of fibronectin with tissue transglutaminase. Biochemistry 28:628-635

Wirtschafter ZT (1966) Genesis of the mouse skeleton. CH. C. Thomas, Springfield, pp 18-20 